\title{
BMJ Open Associations between highly active antiretroviral therapy and the presence of HPV, premalignant and malignant cervical lesions in sub-Saharan Africa, a systematic review: current evidence and directions for future research
}

\author{
Sonia Menon, ${ }^{1,2}$ Rodolfo Rossi, ${ }^{3}$ Natasha Zdraveska, ${ }^{4}$ Mbabazi Kariisa, ${ }^{2}$ \\ Sushama D Acharya, ${ }^{2}$ Davy Vanden Broeck, ${ }^{1,5}$ Steven Callens ${ }^{6}$
}

To cite: Menon S, Rossi R, Zdraveska N, et al. Associations between highly active antiretroviral therapy and the presence of HPV, premalignant and malignant cervical lesions in sub-Saharan Africa, a systematic review: current evidence and directions for future research. BMJ Open 2017;7:e015123. doi:10.1136/ bmjopen-2016-015123

- Prepublication history and additional material are available. To view these files please visit the journal online (http://dx.doi. org/bmjopen-2016-015123)

SM and RR contributed equally.

Received 10 November 2016 Revised 10 April 2017 Accepted 10 April 2017

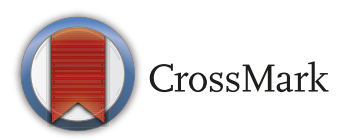

For numbered affiliations see end of article.

Correspondence to

Rodolfo Rossi;

rossirodolfo@yahoo.co.uk

\section{ABSTRACT}

Objectives In sub-Saharan Africa, substantial international funding along with evidence-based clinical practice have resulted in an unparalleled scale-up of access to antiretroviral treatment at a higher CD4 count. The role and timing of highly active antiretroviral therapy (HAART) in mediating cervical disease remains unclear. The aim of this article is to systematically review all evidence pertaining to Africa and identify research gaps regarding the epidemiological association between HAART use and the presence of premalignant/malignant cervical lesions. Method Five databases were searched until January 2017 to retrieve relevant literature from sub-Saharan Africa. Publications were included if they addressed prevalence, incidence or clearance of human papillomavirus (HPV) infection in women undergoing HAART as well as cytological or histological neoplastic abnormalities. Results 22 studies were included, of which seven were prospective studies. Women receiving HAART are less likely to develop squamous intraepithelial lesions (SILs). There is evidence that duration of HAART along with the CD4 count may reduce the prevalence of high-risk HPV (HR-HPV), suggesting that without HAART, severe immunosuppression increases the risk of becoming or remaining infected with HR-HPV. Furthermore, according to existent literature, the CD4 count, rather than HAART coverage or its duration, plays a central role in the prevalence of cervical intraepithelial neoplasia (CIN) 2 and CIN 3.

Conclusion Our findings suggest a positive impact of HAART duration, in conjunction and interaction with CD4 count, on reducing the prevalence of HR-HPV. The greatest treatment effect might be seen among women starting at the lowest CD4 count, which may have a more instrumental role in cervical oncogenesis than either HAART use or the treatment duration on the prevalence of CIN 2 and CIN 3. There is still insufficient evidence to show a clear association between HAART coverage and the incidence of invasive cervical cancer. Enhanced surveillance on the impact of HAART treatment is crucial.

\section{Strengths and limitations of this study}

- Comprehensive review within five databases on a major public health concern for women's health.

- Contributes to and summarises the evidence of the role of CD4 count and highly active antiretroviral therapy (HAART) use in the progression to cervical disease for women who are HIV-positive in Africa.

- High variability in the sample size of the retrieved studies, therefore difficult to give the right weight to the current evidence.

- Heterogeneity in the sensitivity or specificity of screening methods may also render results less comparable; in some studies, HIV status of participants may not have been accurately measured, leaving the possibility of misclassification.

- Lack of prospective studies, with a clear strategy to deal with the loss to follow-up, retrieved by the literature search using the same definition of regression and progression of cervical disease; therefore the criterion of temporality for causality is not well fulfilled.

\section{INTRODUCTION}

Cervical cancer is the second most common female malignancy and the leading cause of death from cancer in women worldwide, of which $85 \%$ of cases occur in low and middle income countries. ${ }^{1}$ The human papillomavirus (HPV), one of the most common sexually transmitted infections, is now well established as the aetiological agent. 'High-risk' (HR) HPV DNA has been found to be present in $99.7 \%$ of cervical cancers worldwide. ${ }^{2}$ Over $200 \mathrm{HPV}$ genotypes have been identified and are divided into HR and low-risk (LR) carcinogens, depending on their potential to cause cancer. 
Invasive cervical cancer (ICC) and its precursor, cervical intraepithelial neoplasia (CIN), are associated with persistent infection with HR HPV genotypes. Products of HPV oncogenes E6 and E7 alter normal genetic and cellular functions and induce malignant transformation. HPV infections are usually transient, and even those that persist for a few months are usually cleared naturally, however women infected with HIV are at higher risk of contracting HPV and developing squamous intraepithelial lesions (SILs), the precursor of cervical cancer. ${ }^{34}$ In this population, this type of lesion tends to be more aggressive, persistent and more likely to recur following cervical disease treatment. ${ }^{56}$

In sub-Saharan Africa, the region with the highest prevalence of HIV one and HIV two in the world, the current standard recommendations for first-line adult antiretroviral therapy include two nucleoside reverse transcriptase inhibitors (NRTIs) and one non-nucleoside reverse transcriptase inhibitor (NNRTI) ${ }^{7}$ Protease inhibitors (PIs) are primarily reserved for second-line treatment due to issues of cost, dosing frequency, drug-drug interactions, potential for long-term side effects and higher pill burden. Persons not responding to first-line regimens are usually switched to a cocktail of two NRTIs plus a boosted PI. ${ }^{8}$

While the use of HAART decreased the risk of Kaposi sarcoma and non-Hodgkin's lymphoma, both AIDS-induced cancers, ${ }^{9}$ there was no significant change in the incidence of cervical cancer. ${ }^{10-12}$ Moreover, studies on the impact of HAART on the natural history of cervical SILs have yielded inconsistent results. ${ }^{13}{ }^{14}$ Moreover, most studies have been conducted in industrialised settings and not in sub-Saharan Africa, the area hardest hit by the global HIV/AIDS pandemic, home to nearly 25 million people living with HIV/AIDS. ${ }^{15}$

While the use of HAART might be expected to reduce the incidence of cervical cancer and progression of cervical dysplasia as a result of improved immunological function, as women are accessing HAART, their life expectancy will increase, due to less competing risk of dying from an opportunistic infection, which in turn may put them more at risk for ICC. ${ }^{16}$ These two reasons will augment their risks of a premalignant condition to transform into an ICC. Inversely, prolonged use of HAART might potentially prevent the acquisition of HR-HPV and/or evolution to ICC.

In the HAART policy landscape, there have been recent shifts towards earlier initiation of treatment. Until 2009, the WHO recommendations for sub-Saharan Africa stipulated initiation of antiretroviral treatment at a CD4 count threshold of 200 cells $/ \mathrm{mm}^{3}$ in order to further reduce the risk of HIV transmission to others. However, this guideline was revised in 2009 and initiation of antiretroviral therapy recommended at CD4 counts of 350 cells $/ \mathrm{mm}^{3}$, only to be revised in 2013 and the treatment threshold set to 500 cells $/ \mathrm{mm}^{3}$ or less. ${ }^{17}$ The policy was again recently revised and treatment initiation recommended for all adults with HIV infection adults, regardless of WHO clinical stage or CD4 cell count, following the results of the START trial. ${ }^{18}$

As a research gap, it remains to be determined whether HAART could improve control of and reduce the burden of HPV infection and the associated precancerous states if initiated even earlier in the course of HIV infection. ${ }^{19}$ The role and timing of HAART in mediating this relationship has been difficult to study and remains unclear. ${ }^{2021}$ The objectives of this paper are to review the existing evidences on the association between the use of HAART and presence of HPV infection and premalignant/malignant cervical lesions.

\section{METHODOLOGY}

We conducted this systematic review based on a pre-defined search protocol that conformed to the criteria set out by the PRISMA statement (see Research Checklist). ${ }^{22}$ CINAHL, SCOPUS, Global Health, PubMed and Embase databases were searched (figure 1).

\section{Search terms}

The domains of the search terms were antiretroviral therapy, human papillomavirus, abnormal cytology, cervical dysplasia, cervical cancer and sub-Saharan Africa (see online supplementary file 1).

\section{Inclusion criteria}

Article selection criteria included any clinic-based randomised controlled trials (RCTs), meta-analyses/ systematic reviews, observational or population-based linkage studies documenting both HAART status and $\mathrm{HPV} / \mathrm{CIN} / \mathrm{ICC}$ rates in sub-Saharan Africa. The five databases mentioned above were searched in January 2017 for articles published from January 1997 onwards to limit the publications to the combined HAART era. The following types of articles were excluded from our search: case reports, articles that did not include combination antiretroviral therapy, risk factors for CIN 2+ or did not stratify data by HAART users. We limited our search to English language literature.

\section{Study selection}

We considered the following four components (Population, Intervention, Comparison, Outcome-PICO) to assess and categorise studies to be included in this review.

Population: the population eligible for this study includes women with HIV infection or women with AIDS with concomitant HPV infection and/or with various degrees of abnormal cytology diagnosed through a recognised method such as PCR HPV genotyping, cytology, visual inspection with acetic acid (VIA), visual inspection with Lugol's iodine (VILI) and histologically confirmed biopsies, and living in sub-Saharan countries.

Intervention: the intervention examined in this study is the administration of HAART, defined as at least three antiretroviral drugs belonging to two drug classes (NRTI, NNRTI or PI) and (if possible) the duration of treatment.

Comparison: comparison is made between women not receiving HAART and women receiving HAART. 

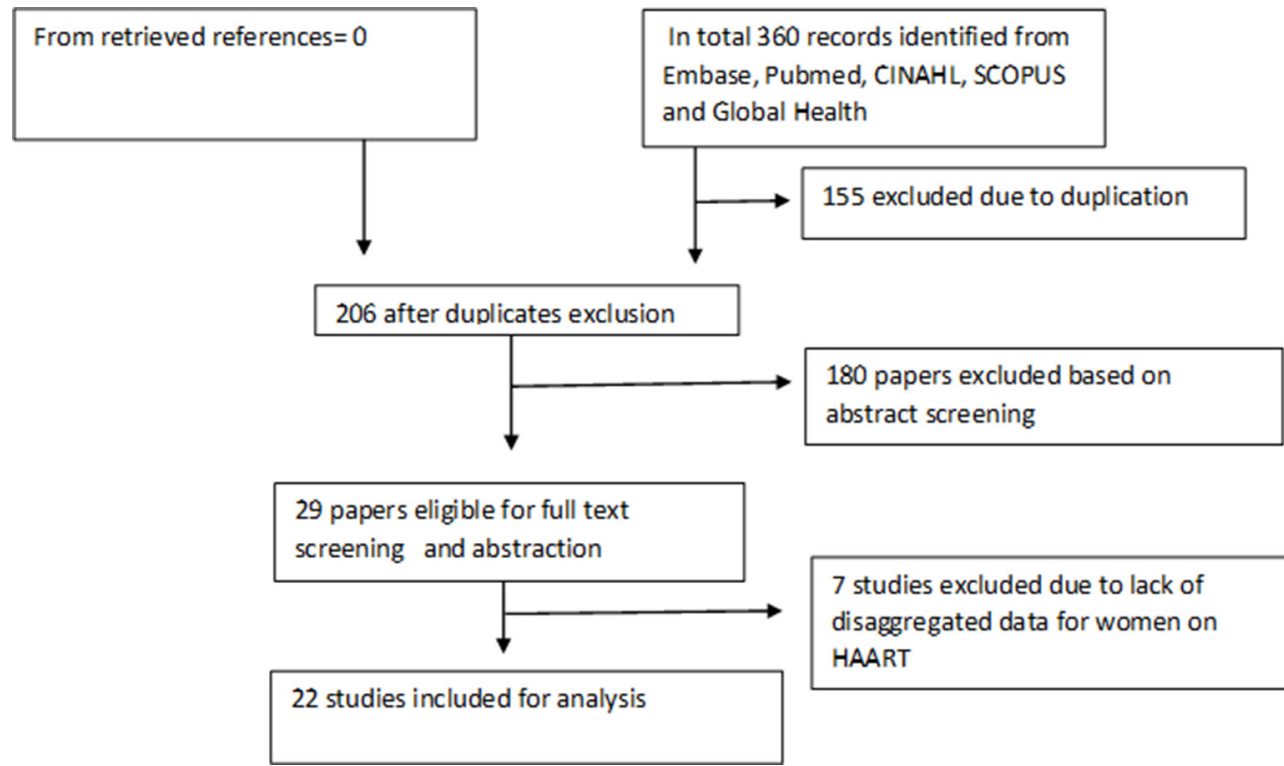

7 studies excluded due to lack of disaggregated data for women on HAART

Figure 1 Flowchart with steps to retrieve the studies to include in the review.

Outcome(s): The wide spectrum of cervical diseases, ranging from the HPV infection (and its potential clearing) through cervical oncogenesis and precursor lesions at the lower end of the neoplastic spectrum, to invasive cervical dysplasia.

All potentially relevant publications were evaluated by two reviewers (SM and RR) and were included in this review if they addressed any of the following outcomes: (1) prevalence, incidence of onset or clearance of HPV infection in women on HAART; (2) prevalence of cervical cytological or histological neoplastic abnormalities or ICG in HAART users. Possible cases of disagreement about the inclusion or exclusion of articles were resolved on an individual basis via thorough discussion among the authors of the justification for exclusion/inclusion.

With the exception of steps concerning the quantitative synthesis (meta-analysis), the PRISMA 2009 checklist and flow diagram ${ }^{23}$ were used to screen and include studies. We also enriched the search by reviewing reference lists from retrieved publications to identify additional manuscripts not captured by the searches. In reporting the results, we used the original terminology as stated in the original articles.

Duplication removal: the original searches from all the databases were combined in an EndNote library (EndNote version 8, San Francisco, California, USA) and all duplicates removed.

\section{Extraction of relevant information from the selected studies}

For all publications, SM and RR recorded the following variables: study location, year of study, study design, study population, main exposure of interest, main outcome of interest, confounding factors adjusted for and main findings.

\section{Data assessment}

Because of the lack of randomised trials retrieved (due to ethical considerations), we considered prospective/ longitudinal studies of the highest quality. We reported an overall score (from 3 to 1 ) based on our assessment of the overall quality of evidence for the following parameters: (1) minimisation of selection bias: (score ' 3 '= strong) the participants were likely, (score '2'= moderate) somewhat likely, (score ' 1 ' = weak) not likely to represent the target population. (2) Study design: (score '3' = strong) prospective study and large sample size (higher than 200) ${ }^{23}$; a prospective study with a sample size lower than 200 and a cross-sectional study with a sample size $>200$ (score '2' = moderate); and a cross-sectional study with a sample size under 200 (score ' 1 ' = weak). (3) Diagnostic accuracy: (score '3') strong histopathological diagnosis; moderate (score '2') VIA/VILI using colposcopy with biopsy solely in cases when lesions appeared suspicious for CIN; and weak (score ' 1 ') cytological diagnosis. (4) Confounders: (score '3') strong, controlled for all relevant confounders; (score '2') moderate, controlled for some confounders; (score ' 1 ') weak, control for confounders not specified. (5) Withdrawals, loss to follow-up and dropouts: (score ' 3 ') strong, description of a strategy to minimise the loss to follow-up, reported withdrawals and dropouts $(80-100 \%$ of participants completed the study) ${ }^{24}$; (score '2') moderate, reported withdrawals and dropouts; (60\% of participants completed the study); (score ' 1 ') weak, withdrawal and dropout rates not specified.

We assessed the methodological quality of evidence by calculating the mean of each study and attributing the mean one of the qualitative attributes, strong, moderate, and weak.

\section{Results}

On 15 January 2017, we included 22 studies in this review (figure 1). No disagreement appeared between the two main coauthors about the studies to be included. A summary of the study is presented in table 1 . 
Table 1 Summary of the quality of evidence assessment for the included studies

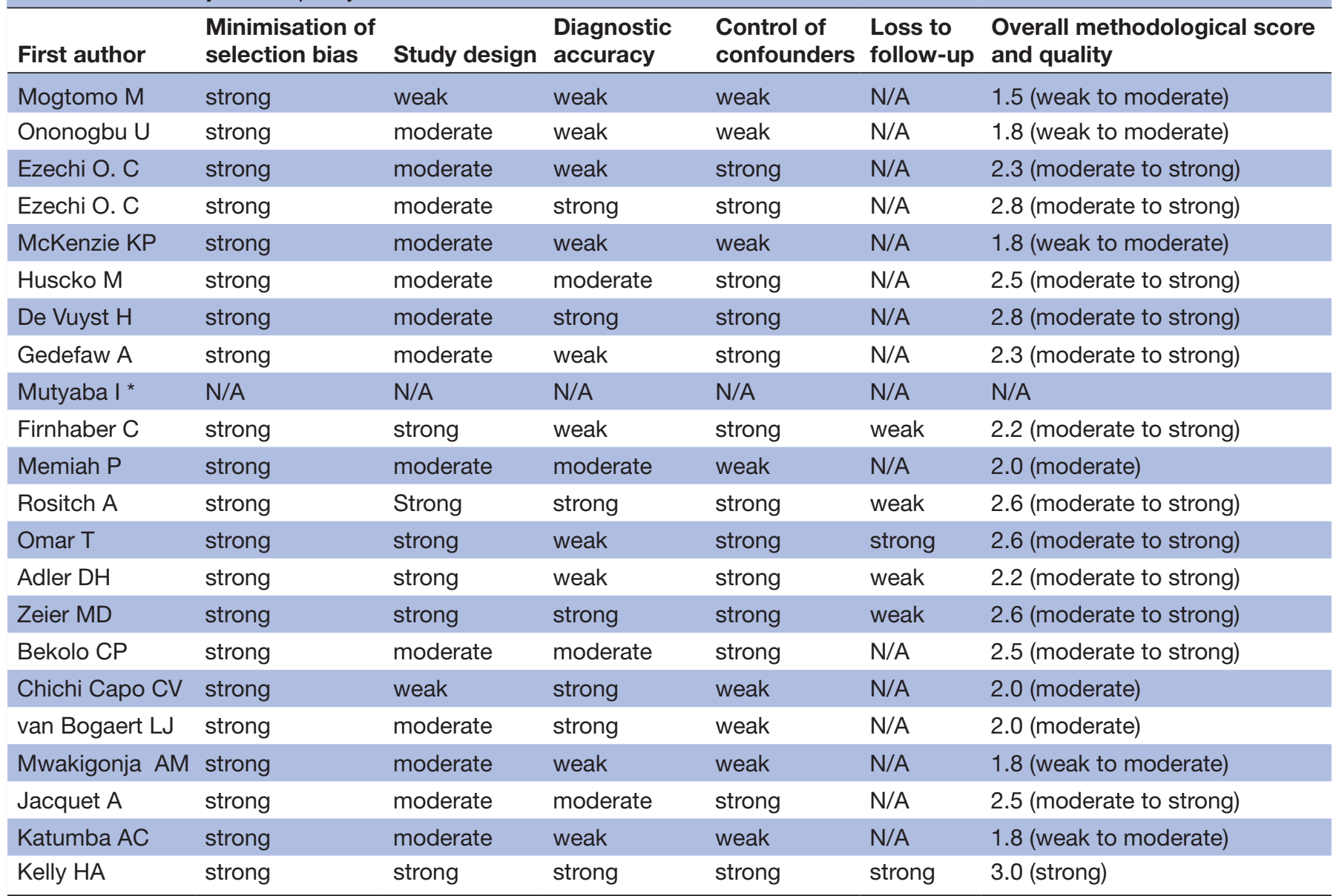

${ }^{\star}$ Ecological study, not amenable to quality assessment since this study is population based and not individual based.

\section{Study population}

A total of 19345 women with HIV infection were included in the studies retrieved and included in this review (one study was an ecological study which did not report the individual sample size). The sample sizes for these studies ranged from 70 to 3241 women with HIV infection.

\section{Study design}

The published literature on HAART and HR HPV presence and cervical dysplasia is characterised by a lack of standardisation in study designs. Twelve studies were cross sectional, two case-control, seven were prospective cohorts and one was a time-series ecological study.

\section{Geographical location}

Two studies were conducted in Nigeria; four in Kenya; seven in South Africa; one in Ethiopia; two in Uganda; two in Cameroon; one in Benin; one in Tanzania; one in Ivory Coast; and one in Burkina Faso (see table 2).

\section{Quality assessment of studies}

The overall quality of evidence ranged from weak/ moderate (score of 1.5) to strong (score of 3) (table 1). Five studies had an average score below 2 and the quality of their evidence was considered as 'weak to moderate'; 15 studies had a score above 2 but less than 3 and the quality of their evidence was considered as 'moderate' or 'moderate to strong. Just one study had a full score of 3 and was considered as 'strong'. One study was ecological in design and could not be assessed in the same way as the other studies that analysed individual data. The quality of evidence was mostly downgraded due to the cross-sectional nature of the studies as well as the lack of studies where samples have been histopathologically confirmed.

We discuss these differences by stage of disease: (1) HAART and HR HPV infection; (2) HAART and SIL/ CIN; (3) HAART and ICC, and outline trends and differences.

\section{Summary of studies on the epidemiological association} between HAART and HR HPV infection/prevalence

Four studies found a significant and inverted association between HAART use and HR HPV infection/prevalence. De Vuyst et al (Kenya, 2012) ${ }^{25}$ reported that the prevalence of HR HPV linearly decreased with use and duration of HAART $(p=0.011)$. This association becomes more evident when this analysis is stratified by CD4 count categories. While the prevalence of HR HPV among patients not receiving HAART linearly increased with each decrease in CD4 count category (CD4 <200; CD4 200-349; CD4 350-499; and CD4 2500; PR for linear trend $5.03, \mathrm{p}=0.025)$, this trend was only marginally significant 
among women receiving HAART for $<2$ years (prevalence ratio (PR) for linear trend 2.98, $\mathrm{p}=0.084$ ) and insignificant for women on HAART therapy for $\geq 2$ years (PR for linear trend 1.19, $\mathrm{p}=0.275$ ). Zejer et al (South Africa $2015)^{26}$ reported a significant reduction in risk of HPV in women on HAART (OR $0.23,95 \%$ CI 0.15 to 0.37 ) in the 204 patients eligible for HAART, and for every additional month of HAART since initiation, the risk of detection of any HPV type decreased by $9 \%$.

Kelly et al (Burkina Faso and South Africa, 2017) ${ }^{27}$ observed that, compared with long-duration antiretroviral therapy users ( $>2$ years), HR HPV prevalence was higher among those on short duration HAART in both countries. However, when adjusted for CD4 cell count, this association was observed in Burkina Faso only (65.1 vs $52.1 \%$ for $<2$ years compared with $>2$ years; aPR $1 / 41.24$, $95 \%$ CI 1.04 to 1.47). Ezechi et al (Nigeria, 2014) reported lower HR HPV prevalence in women who were HIV positive on antiretroviral drugs ( $\mathrm{OR}=0.4 ; 95 \% \mathrm{CI}: 0.3$ to 0.5$)$.

Whereas four studies found a significant epidemiological association between HAART and HR HPV genotypes, one did not. Rositch et al. (Uganda, 2013) ${ }^{28}$ did not find any impact on monthly HPV DNA detection (PR 1.0; $95 \%$ CI 0.96 to 1.08 ), regardless of immune reconstitution or HIV viral suppression. Almost all test subjects (92\%) had detectable HPV in the 6 months preceding HAART initiation and the cumulative prevalence remained high following initiation of therapy (90\%) after a follow-up of 6 months (table 2).

The review revealed heterogeneity between studies in the use of the screening/diagnostic methods for cervical pathology (table 3). Nine studies used cytological changes with reporting based on the revised Bethesda classification, two studies used histopathological confirmation, and five studies used VIA/VILL screening methods of which three ascertained disease using colposcopy with biopsy solely in cases when lesions appeared suspicious for CIN.

Seven cross-sectional studies reported on the frequency of SIL and risk factors for SIL. Memiah et al. (Kenya, 2012) ${ }^{29}$ observed that patients not receiving HAART treatment were 2.2 times more likely to have precancerous lesions than patients receiving HAART (adjusted $\mathrm{OR}=2.21,95 \%$ CI 1.28 to 3.83). Similarly, Mogtomo et al (Cameroon, 2009) ${ }^{30}$ reported that, of 22 high-grade SIL (HSIL)-positive women included in the study, 63.6\% $(14 / 22)$ were not on HAART, whereas $36.4 \%$ (8/22) were receiving HAART. The study revealed that total cervical abnormalities (HSIL and low-grade SIL (LSIL)) had a prevalence of $48.6 \%(\mathrm{n}=17)$ among HAART users compared with $62.9 \%$ among women not receiving HAART $(\mathrm{p}=0.034)$.

In line with the association between the absence of HAART treatment and development of SIL, O. Ezechi et al (Nigeria, 2013) showed an increased risk of both SIL (adjusted OR=2.0; 95\% CI 1.2 to 3.5) and HSIL (adjusted OR=2.6; 95\% CI 1.0 to 6.7$)^{31}$ among 1140 participants not on HAART, adjusted for age, marital status, age at first intercourse and lifetime sexual partners. Nevertheless, Ononogbu et al (Nigeria, 2013) found only marginal or insignificant associations with different HAART regimens. ${ }^{32}$ Their study reported that 2501 women on tenofovir-containing HAART had a borderline significant RR of $1.4(95 \%$ CI 1.0 to 2.0), and a RR of 1.5 (95\% CI 1.0 to 2.2) on zidovudine-containing HAART, whereas women on stavudine-containing HAART showed a RR of 0.9 (95\% CI 0.4 to 2.11) of having cervical disease compared with women not receiving HAART. ${ }^{29}$

Hushko et al (Kenya, 2014) ${ }^{33}$ observed that combined oral contraceptives remained significantly associated with detection of CIN 2+ in women on HAART (adjusted $\mathrm{OR}=1.84 ; 95 \%$ CI 1.20 to 2.82 ), compared with in women not HAART (adjusted OR=1.72; 95\% CI 1.08 to 2.73), whereas the use of a progesterone implant was associated with increased detection of CIN 2+ (adjusted OR=9.43; $95 \%$ CI 2.85 to 31.20 ) only among women not on HAART. McKenzie et al (Kenya, 2011) ${ }^{34}$, who explored risk factors for SIL development in women on HAART, reported no significant difference in the use of neviparine-based regimens compared with efavirenz-based regimens between women with normal cytology and those with SIL ( $74 \%$ vs $72 \% ; \mathrm{p}=0.80$ ). Similarly, there was no difference in the prevalence of nucleoside reverse transcriptase inhibitors backbones containing zidovudine compared with those containing stavudine between women with SIL and those without $(12 \%$ vs $15 \%$; $\mathrm{p}=0.41)$.

Bekolo et al (Cameroon, 2016) ${ }^{35}$ also reported a decreased risk of cervical lesions among HAART receivers. After controlling for age and other covariates, women in the HAART group had a $67 \%$ reduction in the odds of cervical lesions compared with the community group (adjusted OR=0.33; 95\% CI 0.15 to $0.73 ; \mathrm{p}=0.006$ ).

Mwakigonja et al (Tanzania, 2012) ${ }^{36}$ identified immunodeficiency as the main determinant of the presence of CIN+ in HIV-infected women: CD4 count $>350$ cells/ $\mathrm{mm}^{3}(\mathrm{OR}=0.3 ; 95 \%$ CI 0.2 to 0.6$)$ or $\geq 200-350$ cells $/ \mathrm{mm}^{3}$ (OR=0.6; 95\% CI 0.4 to 1.0$)$ (Ref: $<200$ cells $\left./ \mathrm{mm}^{3} \mathrm{CD} 4\right)$.

One study, Katumba et al (South Africa, 2010) reported that women who did not use HAART had more abnormal results compared with those who used HAART $(\mathrm{p}<0.028)$, although no confounders were adjusted for.

Two longitudinal studies reporting on progression/ regression of cervical disease in women with HIV infection on HAART were identified. There was a lack of harmony in how regression/ progression was considered. A prospective observational study conducted in South Africa by Firnhaber et al (2010) ${ }^{37}$ suggested that HAART was associated with a robust reduction in the incidence and progression of cervical disease (adjusted IRR $=0.55 ; 95 \%$ CL 0.37 to 0.80 ). The study sensitivity analyses confirmed that the result was not dependent on the length of HAART exposure and this significant effect of HAART was only seen in women with CD4 counts of $<350$ cells $/ \mathrm{mm}^{3}{ }^{34}$ ASC-US (atypical squamous cells of undetermined significance) results were classified as 


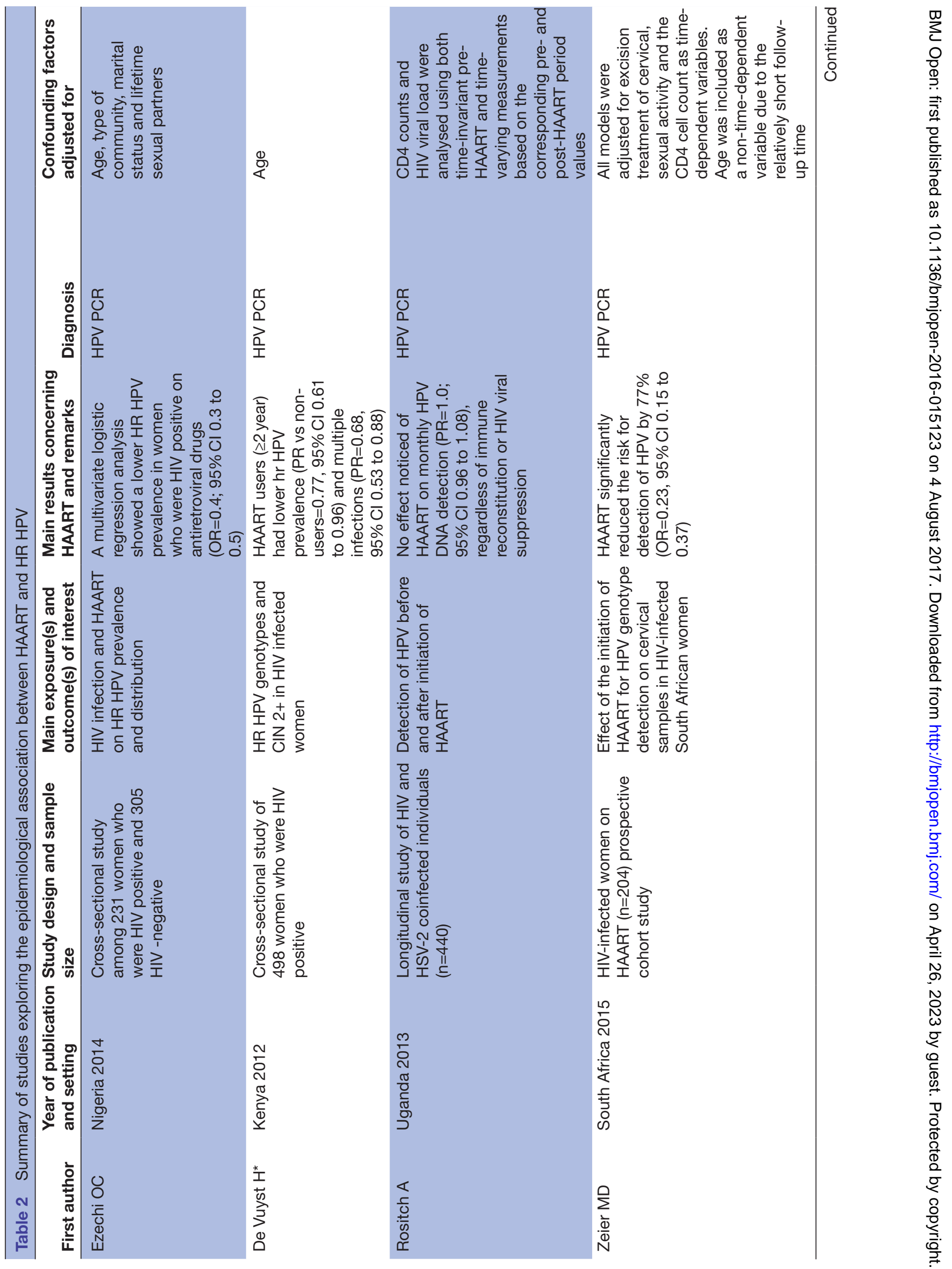




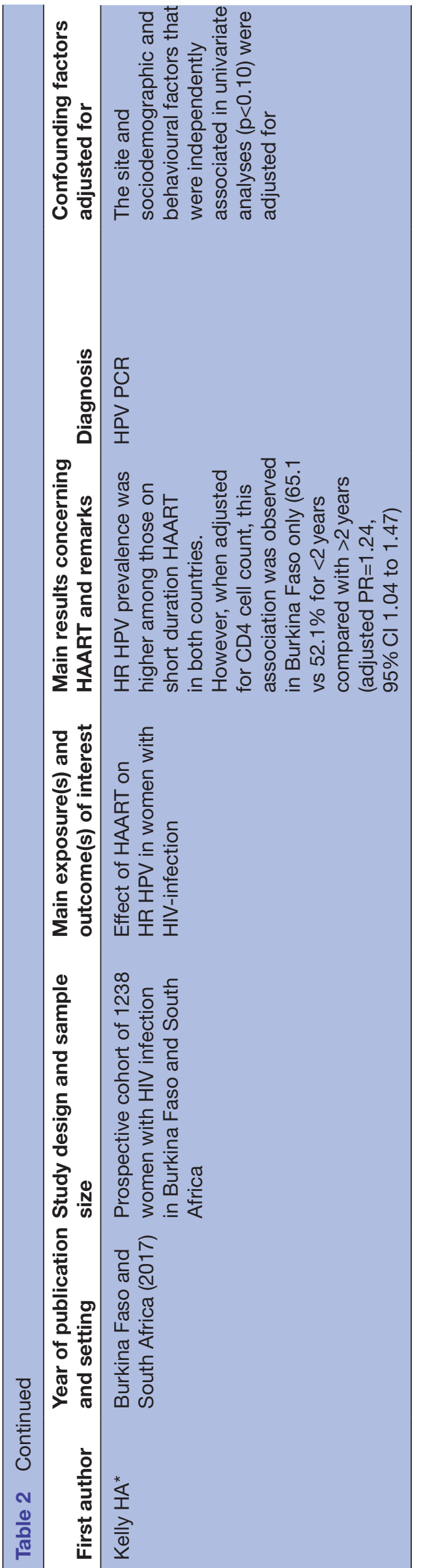

LSIL results, while ASC-H results were classified as HSIL results.

A smaller magnitude of effect was found by Omar et al (South Africa, 2011) ${ }^{38}$ (HR=0.72; 95\% CL 0.52 to 0.99) using time-varying CD4 counts as a covariate in the multivariate model of progression. Progression was defined either as a subsequent smear with a cytological diagnosis of ASCH, HSIL or worse in women who had a previous normal or LSIL smear with an interval between smears of $>5.5$ months.

One study was identified on HAART and regression of cervical disease. A large prospective cohort study from Soweto, South Africa undertaken by Adler et al (2012) ${ }^{39}$ reported that HAART positively affected the natural history of cervical disease in women with HIV infection and a multivariate marginal models analysis identified a significantly increased likelihood (OR=2.61; 95\% CI 1.75 to $3.89 ; \mathrm{p}<0.0001$ ) of regression of cervical lesions among women on HAART. Regression was defined as a subsequent improvement in cytological results from normal, ASC-US, LSIL, ASC-H, HSIL and cancer.

Other studies reported specifically on the duration of HAART and SIL. Kelly et al (South Africa, 2017) ${ }^{27}$ reported that CIN 2+ prevalence was higher among short-duration antitretroviral therapy users $<2$ years (adjusted $\mathrm{OR}=1.99$; 95\% CI 1.12 to 3.54) and naive participants (adjusted OR=1.87; 95\% CI 1.11 to 3.17) in South Africa. De Vuyst et al (Kenya, 2012) ${ }^{25}$ reported that the prevalence of CIN 2 and CIN 3 did not vary across HAART non-users, HAART users for $<2$ years or $\geq 2$ years ( $p$ for linear trend $=0.416$ ). However, when stratified for CD4 count categories, the prevalence of CIN 2/3 among HAART non-users linearly increased per each decrease of CD4 count category, CD $4<200$; CD4 200-349; CD4 350-499; and CD4 $\geq 500$ (p for linear trend $=0.013$ ) but this is not seen among women receiving HAART for $<2$ years and $\geq 2$ years ( $p$ for linear trend $=0.9$ and 0.5 , respectively). Still, CD4 nadir before initiating HAART was unknown and HAART may have been started at a CD4 count too low to prevent or reverse CIN 2/3. Similarly, Huchko et al (Kenya, 2014) ${ }^{30}$ detected a non-significant association between the duration of HAART and CIN 2+ (adjusted OR $=0.98 ; 95 \%$ CI 0.95 to 1.00 ), although it was not adjusted for CD4 count. McKenzie (Kenya, 2011) ${ }^{31} \mathrm{did}$ not find any significant correlation between the duration and type of HAART and SIL and the median number of months on HAART did not differ between women with normal cytology and those with SIL (14 vs 11 months; $\mathrm{p}=0.17$ ).

Nevertheless, Mogtomo et al (Cameroon, 2009) ${ }^{30}$ observed that while LSIL incidence in wowmen with HIV infectionunder HAART therapy decreased from 8.6\% (three cases) to $5.7 \%$ (two cases) the first 10 months of HAART use, it increased to $11.4 \%$ (four cases) after 10 months. In contrast, HSIL cases decreased from $14.3 \%$ $(\mathrm{n}=5)$ to $5.7 \%$ to $2.8 \%(\mathrm{n}=1)$ after 10 months.

Capo Chichi et al $(2016)^{40}$ in Benin reported that cervical dysplasia was observed in 4/86 (5\%) of women living with HIV on HAART, in whom after 2 years no new 
cases of cervical dysplasia were reported, nor were the HPV genotypes present at baseline there after 2 years.

A cross-sectional study from Ethiopia (Gedefaw et al 2013) showed that the presence of precancerous lesions was inversely associated with HAART: patients under HAART at the moment of the study (not specifying the duration of the treatment) were $48 \%$ less likely to have precancerous cervical cancer lesions than those who were not on HAART (adjusted OR=0.52, 95\% CI 0.35 to 0.92 ; $\mathrm{p}=0.015){ }^{41}$

One prospective study assessed CIN 2+ incidence. Kelly et al (South Africa, 2017) ${ }^{27}$ reported that CIN 2+ incidence was reduced among women on HAART (adjusted $\mathrm{O}=0.39 ; 95 \%$ CI 0.15 to 1.01 ).

Four studies contrasted in their reported results concerning the protective effect of higher CD4+ count nadir, prior to HAART initiation, on the onset of cervical disease. Huchko et al (Kenya, 2014) ${ }^{30}$ reported that CD4+ nadir over 500 cells $/ \mathrm{mm}^{3}$ was associated with a reduced detection of CIN 2+ (adjusted OR=0.61, 95\% CI 0.38 to 0.97 ) in the overall group, but current $\mathrm{CD} 4+$ was only associated with reduced detection of CIN 2+ among HAART non-users (adjusted OR=0.42; 95\% CI 0.22 to 0.80$).{ }^{42}$ This finding is compatible with the immune driven trend between $\mathrm{CD} 4+$ and a positive cervical screening among women with HIV infection observed by Ononogbu et al (Nigeria, 2013) ${ }^{43}$ which reported a decreasing risk with increasing CD4 count: $\mathrm{RR}=0.5$ (95\% CI 0.3 to 0.7 ) for CD4 count 300 to $<450 ; \mathrm{RR}=0.5$ (95\% CI 0.3 to 0.7 ) for 450 to $<650$; and $\mathrm{RR}=0.3$ (95\% CI 0.2 to 0.6 ) for $\geq 650$.

A prospective observational study of 601 HIV-seropositive women conducted by Firnhaber et al (South Africa, 2012) suggested that the effect of baseline HAART exposure was strongly modified by baseline $\mathrm{CD} 4$ count when initiating HAART in women with $\mathrm{CD} 4$ counts $<350$ cells/ $\mathrm{mm}^{3}$. A RR=0.47 (95\% CI 0.30 to 0.73 ) was found in women initiating HAART with CD4 counts $<350$ cells/ $\mathrm{mm}^{3}$ compared with $\mathrm{RR}=0.84(95 \%$ CI 0.42 to 1.72$)$ in women with baseline CD4 counts $\geq 350$ cells $/ \mathrm{mm}^{3}$.

Only one study reported baseline CD4 count not to be significant. Jacquet et al (Ivory Coast, 2014) ${ }^{44}$ reported that the baseline $\mathrm{CD} 4$ count at the time of first clinical follow-up, which could be interpreted as a proxy for nadir CD4 count, was not found to be significantly associated with $\mathrm{CIN}+$ in the multivariate model (table 3 ).

\section{Epidemiological association between HAART and ICC}

Only two studies were found that assessed the effect of HAART on cervical cancer (table 3). An age-adjusted population-level evaluation of the effect of antiretroviral therapy on cancer incidence in Kyadondo County, Uganda (1999-2008), revealed that the increase in population-level HAART coverage over time did not affect the incidence of invasive cervical cancer (IRR $=1.02$; $95 \%$ CI 0.98 to 1.05$).{ }^{45}$ However, Bogaert et al (South Africa, 2013) reported that patients on HAART had not only less CIN $1 \quad(\mathrm{p}=0.018)$ and CIN $2(p=0.18)$ but also less ICC $(p=0.019)$ than their untreated counterparts, despite similar mean CD4 count.

\section{DISCUSSION}

The findings of our systematic review suggest a positive impact of HAART duration, in conjunction and interaction with $\mathrm{CD} 4$ count, on reducing the prevalence of HR HPV. Our review also illustrates how untreated people living with HIV with low CD4 count may be at increased risk of being or remaining infected with HR HPV compared with those treated with HAART. Moreover, our findings support the hypothesis that the greatest treatment effect might be seen among women starting at the lowest CD4 count, since higher CD4 count might be associated with lower incidence of CIN 2+, thereby reducing the possible treatment effect of HAART.

The literature identified in this systematic review suggests that CD4 count may have a more instrumental role in cervical oncogenesis or the integration of the latent reservoir throughout the body than either HAART use or the treatment duration on the prevalence of CIN 2 and CIN 3.

Surprisingly, we were unable to retrieve any studies that assess the impact of HAART on cervical disease and HPV infection across different types of HIV infections. It therefore remains unknown whether the effect of HAART differs between patients with HIV-1, HIV-2 or dual infection. HIV-2 infection and dual infection are highly prevalent in sub-Saharan Africa, predominantly in Western Africa in countries like Guinea Bissau, Ivory Coast, Mali and Burkina Faso. ${ }^{46}$

The sample size of the studies included in this review greatly varies, rendering it difficult to assess and compare strength of associations. Due to various difficulties, such as financial and ethical considerations linked to the conduct of RCTs, evidence is mostly based on retrospective or cross-sectional studies, which do not fulfil the causal criterion of temporality. Heterogeneity in the sensitivity or specificity of screening methods may also render results less comparable. Cervical cytology, which has a clinical sensitivity between $55 \%$ and $65 \%$ for detection of histopathologically confirmed 'true disease status' ${ }^{47}$ along with low interobserver or intraobserver true correlation can result in even one grade of misclassification introducing a bias in studies assessing regression or incidence of SIL in women on HAART. Moreover, a recent study in Kenya has reported that the sensitivity of VIA is affected in women on HAART and by CD4 count, ${ }^{48}$ which in studies ascertaining disease using colposcopy with biopsy solely in cases when lesions appeared suspicious for CIN 2+, may have led to under-ascertainment of true disease: sensitivity $89.9 \%$ (95\% CI $82.2 \%$ to $95.0 \%$ ) in women under 35 and $78.6 \%$ (95\% CI $63.2 \%$ to $89.7 \%$ ) in women above $35 .{ }^{47}$

Our findings derived from the sub-Saharan context contrast with the conclusion of a systematic review of studies from industrialised settings suggesting a largely inconsistent impact of HAART on reducing the incidence and progression and facilitating the regression of HPV infection and cervical abnormalities. ${ }^{49}$ This discrepancy may be attributed to the greater access in industrialised 

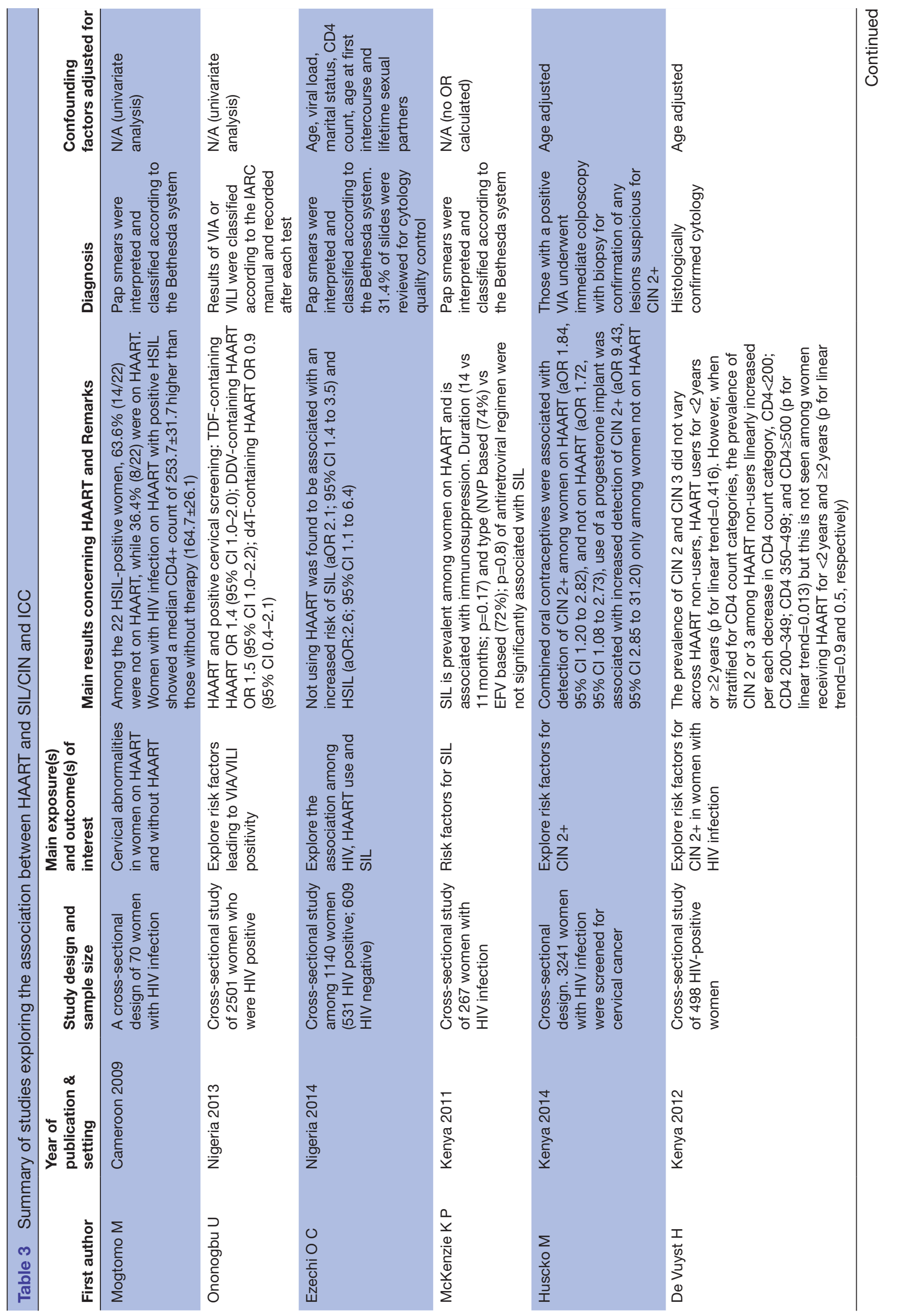


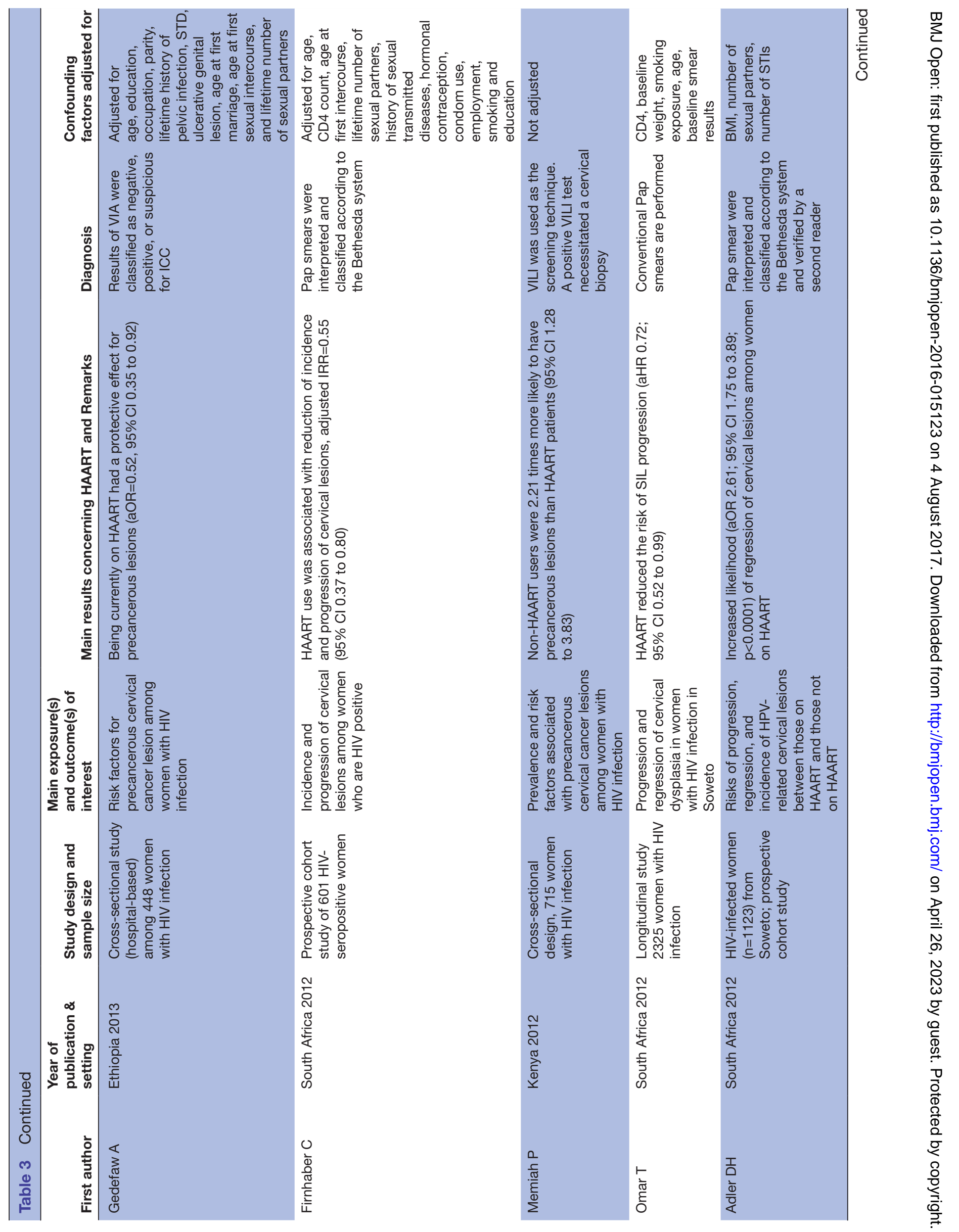




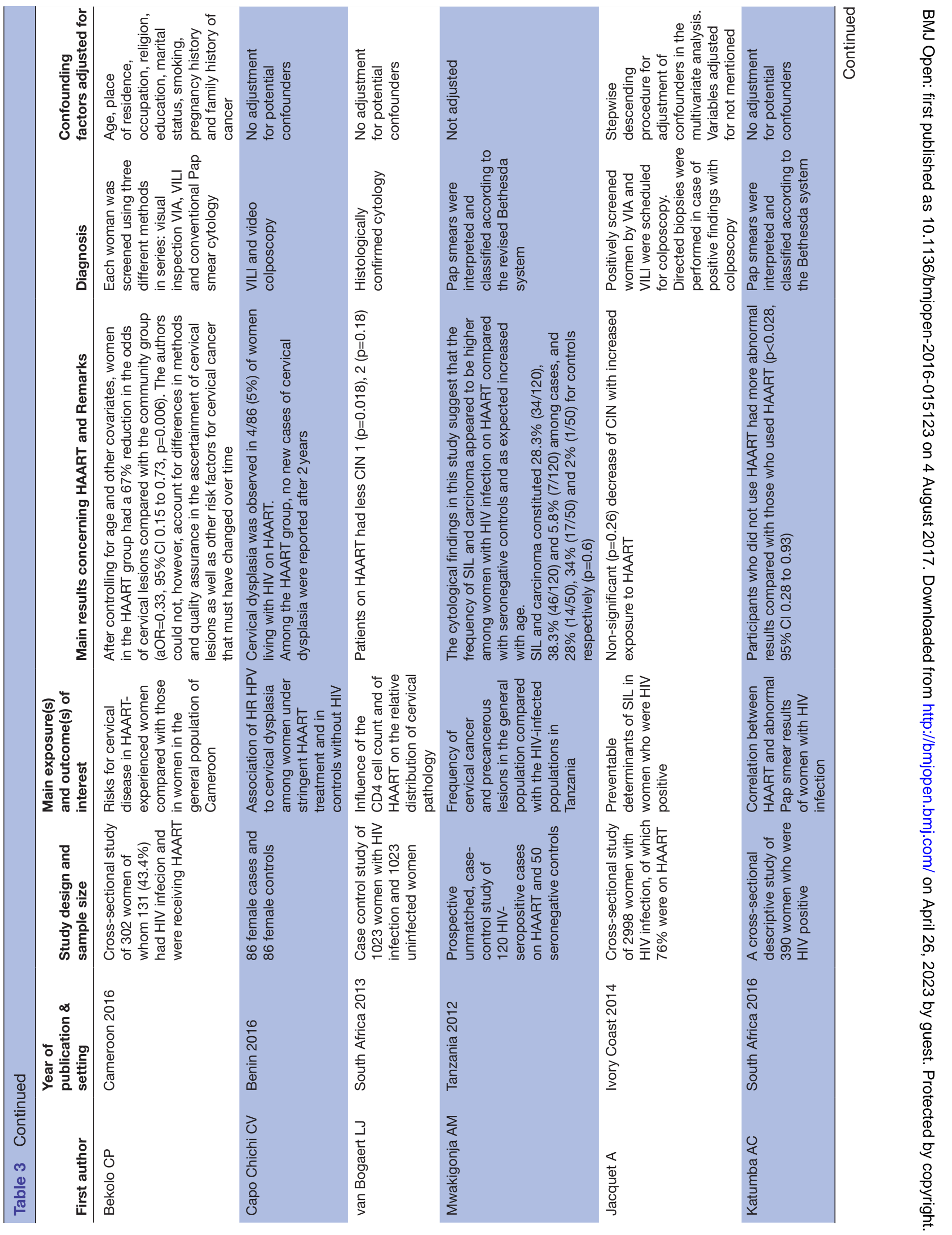




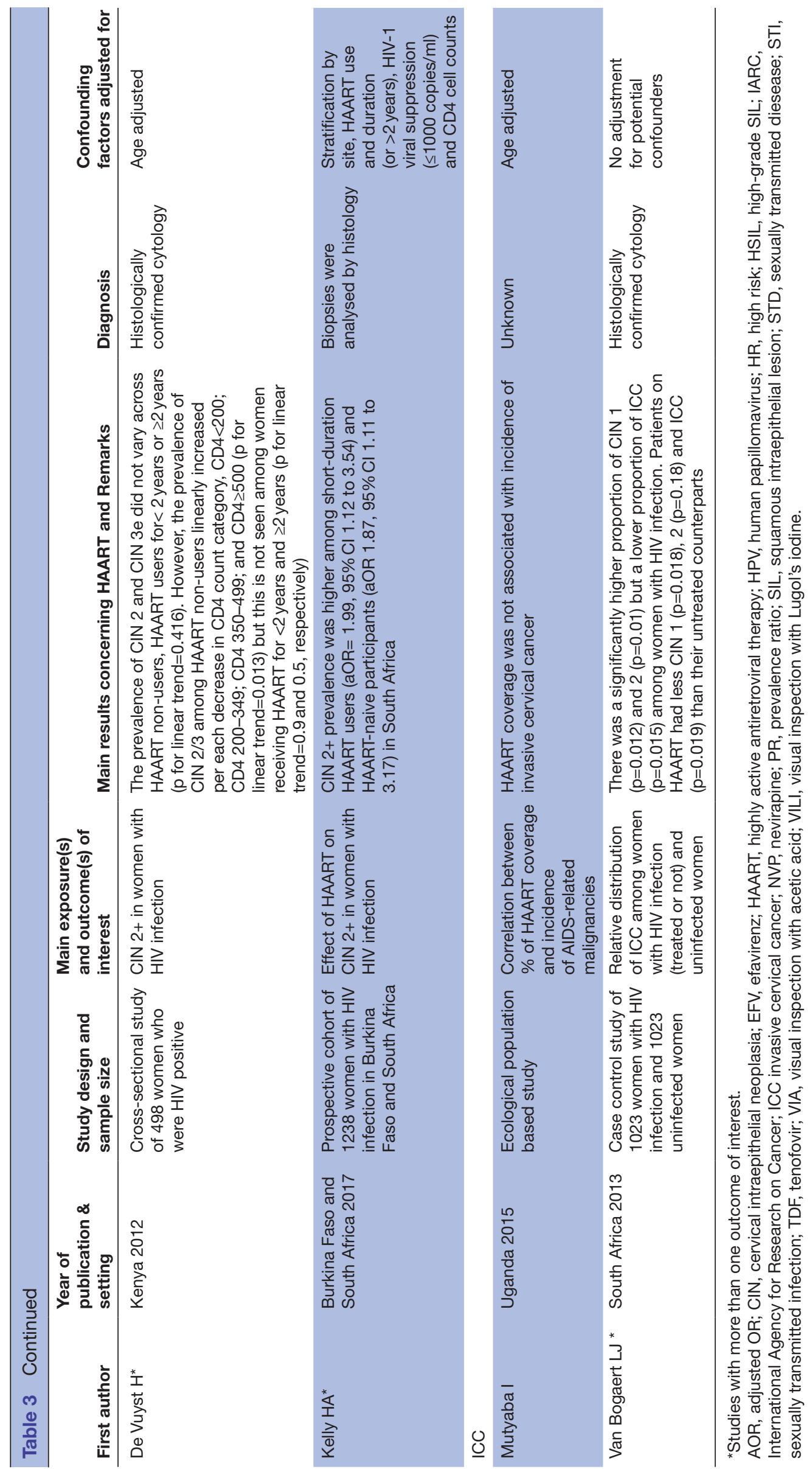


settings to frequent cytological screening, thereby making the potential benefits of HAART less apparent.

One of the few retrieved prospective studies exploring the impact of HAART on SIL incidence and progression only reported a significant association in women with $\mathrm{CD} 4$ counts $<350$ cells $/ \mathrm{mm}^{3}$. Apart from the inclusion criteria providing heterogeneity in baseline profiles of women, different potential confounders have been adjusted for in studies addressing the same outcome. Not all studies control for hormonal/barrier contraception use, HIV viral load, CD4 count baseline or the number of sexual partners, therefore residual confounding cannot be excluded. In addition, the duration of follow-up may be too short and not always detailed in articles.

\section{Clinical and public health implications}

The lack of statistically significant associations observed in this review between duration of HAART intake and reduction of CIN 2+ in women initiating HAART at an earlier $\mathrm{CD} 4$ count initiation suggests the importance of continued follow-up with cervical cytological and/or histological screening. Even after HAART initiation and immune reconstitution, lesions remain prevalent and progression of disease should be studied further to allow best timing of a screening schedule.

Although current HAART guidelines in industrialised countries promote biannual cervical cancer screening in the first year following HIV diagnosis and annually thereafter, in the absence of evidence-based knowledge in sub-Saharan African women, it may be advisable to address the following research gaps before suggesting the relevance of the guideline in this population. In the mean time, a cautionary approach may be employed and biannual cervical screening continued, if feasible.

\section{Research gaps}

The dearth of studies on duration and initiation of HAART at an earlier CD4 count and the consensus on findings preclude policy makers from developing local guidelines. Moreover, due to the changes in recommendations for HAART start, women in sub-Saharan Africa at the time of the studies were more likely to have low CD4 counts, whereas in the future they will be initiating HAART in a far less immunosuppressed state.

Future studies should preferably be prospective in design. This will allow a better follow-up of patients at different points in time and fulfil the criterion of temporality for causality. Moreover, including nested case-control studies within cohort follow-up will allow robust intermediary evidence for such research questions. Studies should also elucidate the association between cervical HIV DNA and HPV incidence/prevalence, or cervical dysplasia.

The exploration of an association between HAART and cervical cancer warrants large cancer epidemiological studies. Previously, the lack of effect of HAART on the prevalence on cervical cancer can be ascribed to the high mortality associated with AIDS. Despite improved longevity, and in settings with a life expectancy that exceeds the average age of cervical cancer onset, one study to date from our review suggested that, thanks to extensive HAART programmes, decrease in cervical cancer incidence can be expected. ${ }^{50}$

Finally, women with HIV-HPV coinfections may be at risk for immune-modulating helminthic, tuberculosis and malarial infections ${ }^{51}$, which may compromise the positive impact that HAART may have on clearing cervical lesions. Their synergistic interactions in the post-HAART era should be elucidated.

\section{CONCLUSION}

Access to HAART in sub-Saharan Africa has dramatically increased over the past decade, improving life expectancy for women living with HIV. This in turn requires urgent clarification of the impact of HAART on the development of cervical precancerous states and cancer.

Whilst the preponderance of studies suggests that women on HAART are less likely to have SIL, for ICC, evidence remains scarce and inconclusive. However, our review imputes a more significant role of $\mathrm{CD} 4$ count or the CD4 count at which HAART is initiated, the timing of HAART in relation to immunosuppression on HR HPV genotype and CIN 2/3 prevalence, than to HAART use, irrespective of its duration. The scarce evidence shows that the positive impact of HAART is only felt in women with $\mathrm{CD} 4$ counts of $<350$ cells $/ \mathrm{mm}^{3}{ }^{3}$.

Given the current limited evidence available to assist policy makers in developing local guidelines and prevention and treatment strategies for cervical cancer among women with HIV infection on HAART, a tailored screening protocol cannot be established. Longer term surveillance data on women who are HIV positive at different levels of immunosuppression is crucial to determine the effect of HAART on CIN $2+$.

Author affiliations

${ }^{1}$ International Centre for Reproductive Health (ICRH), Ghent University, Ghent, Belgium

${ }^{2}$ CDC Foundation, Atlanta, Georgia, USA

${ }^{3}$ Laboratory for Cell Biology \& Histology, University of Antwerp, Antwerp, Belgium ${ }^{4}$ Department of Clinical Pharmacy, Saints Cyril and Methodius, Republic of Macedonia

${ }^{5}$ AMBIOR, Laboratory for Cell Biology \& Histology, University of Antwerp, National Reference Centre for HPV, Laboratory of Molecular Pathology, Antwerp, Belgium ${ }^{6}$ Department of Internal Medicine \& Infectious diseases, University Hospital, Ghent, Belgium

Contributors MS and RR contributed equally to the study: conception of the study, coordination of the study, screening of studies, interpretation of the findings of included studies, writing and editing the paper, validation of the final version. ZN: interpretation of the findings, writing and editing the paper, validation of the final version. KM: editing the paper, validation of the final version. DS: editing the paper, validation of the final version. $\mathrm{BD}$ : editing and validation of the final version. CS: conception, interpretation and validation of the final version.

Competing interests None declared.

Provenance and peer review Not commissioned; externally peer reviewed.

Data sharing statement № additional data are available.

Open Access This is an 0pen Access article distributed in accordance with the Creative Commons Attribution Non Commercial (CC BY-NC 4.0) license, which permits others to distribute, remix, adapt, build upon this work non-commercially, 
and license their derivative works on different terms, provided the original work is properly cited and the use is non-commercial. See: http://creativecommons.org/ licenses/by-nc/4.0/

(c) Article author(s) (or their employer(s) unless otherwise stated in the text of the article) 2017. All rights reserved. No commercial use is permitted unless otherwise expressly granted.

\section{REFERENCES}

1. WHO. Fact sheet: human papilloma and cervical cancer. $2015 \mathrm{http}: / /$ www.who.int/mediacentre/factsheets/fs380/en/ (accessed 17 May 2016).

2. Walboomers JM, Jacobs MV, Manos MM, et al. Human papillomavirus is a necessary cause of invasive cervical Cancer worldwide. J Pathol 1999;189:12-19.

3. Rabkin CS, Biggar RJ, Baptiste MS, et al. Cancer incidence trends in women at high risk of human immunodeficiency virus (HIV) infection. Int J Cancer 1993;55:208-12.

4. Sun XW, Kuhn L, Ellerbrock TV, et al. Human papillomavirus infection in women infected with the human immunodeficiency virus. $N$ Engl $J$ Med 1997;337:1343-9.

5. Ellerbrock TV, Chiasson MA, Bush TJ, et al. Incidence of cervical squamous intraepithelial lesions in HIV-infected women. JAMA 2000;283:1031-7.

6. Rowhani-Rahbar A, Hawes SE, Sow PS, et al. Dembele B critchlow CW, N'doye I, Kiviat NB. the impact of HIV status and type on the clearance of human papillomavirus infection among Senegalese women. J Infect Dis 2007;196:887-94.

7. World Health Organization. Antiretroviral therapy for HIV infection in adults and adolescents: recommendations for a public health approach-2010 revision. http://whqlibdoc.who.int/publications/ 2010/9789241599764_eng.pdf (accessed 15 May 2016).

8. Adult combination antiretroviral therapy in sub-Saharan Africa: lessons from Botswana and future challenges, C William Wester, Hermann Bussmann, John Koethe, Claire Moffat, Sten Vermund, Max Essex, Richard G Marlink. HIV Ther 2009.

9. Pipkin S, Scheer S, Okeigwe I, et al. The effect of HAART and calendar period on Kaposi's sarcoma and non-Hodgkin lymphoma: results of a match between an AIDS and cancer registry. AIDS 2011;25:463-71.

10. Ledergerber B, Telenti A, Egger M. Risk of HIV related Kaposi's sarcoma and non-Hodgkin's lymphoma with potent antiretroviral therapy: prospective cohort study. Swiss HIV Cohort Study. BMJ 1999;319:23-4.

11. Jacobson LP, Yamashita TE, Detels R, et al. Impact of potent antiretroviral therapy on the incidence of kaposi's sarcoma and nonHodgkin's lymphomas among HIV-1-infected individuals. Multicenter AIDS Cohort Study. J Acquir Immune Defic Syndr 1999;21:S34-S41.

12. International Collaboration on HIV and Cancer. Highly active antiretroviral therapy and incidence of Cancer in human immunodeficiency virus-infected adults. J Natl Cancer Inst 2000;92:1823-30.

13. Palefsky JM. Cervical human papillomavirus infection and cervical intraepithelial neoplasia in women positive for human immunodeficiency virus in the era of highly active antiretroviral therapy. Curr Opin Oncol 2003;15:382-8.

14. Heard I, Palefsky JM, Kazatchkine MD. The impact of HIV antiviral therapy on human papillomavirus (HPV) infections and HPV-related diseases. Antivir Ther 2004;9:13-22.

15. Overview HIV/STI. http://www.afro.who.int/en/clusters-aprogrammes/dpc/acquired-immune-deficiency-syndrome/overview. html (accessed 15 May 2016).

16. Adler DH, Kakinami L, Modisenyane T, et al. Increased regression and decreased incidence of human papillomavirus-related cervical lesions among HIV-infected women on HAART. AIDS 2012;26:1645-52.

17. WHO. WHO issues new HIV recommendations calling for earlier treatment. 2013. (accessed 1 Apr 2016).

18. WHO. Guidelines on when to start antiretroviral therapy and on pre exposure prophylaxis for HIV. 2015. http://apps.who.int/iris/ bitstream/10665/186275/1/9789241509565_eng.pdf (accessed 17 May 2016).

19. Palefsky JM. Cervical human papillomavirus infection and cervical intraepithelial neoplasia in women positive for human immunodeficiency virus in the era of highly active antiretroviral therapy. Curr Opin Oncol 2003;15:382-8.

20. Heard I, Potard V, Costagliola D. Limited impact of immunosuppression and HAART on the incidence of cervical squamous intraepithelial lesions in HIV-positive women. Antivir Ther 2006;11:1091-6.

21. Adler DH, Kakinami L, Modisenyane T, et al. Increased regression and decreased incidence of human papillomavirus-related cervical lesions among HIV-infected women on HAART. AIDS 2012;26:1645-52.

22. PRISMA statement. http://www.prisma-statement.org/statement.htm (accessed 2 Jan 2016).

23. BMJ CLinical evidence what is Grade. http://clinicalevidence.bmj. $\mathrm{com} / \mathrm{x} / \mathrm{set} / \mathrm{static} / \mathrm{ebm} / \mathrm{learn} / 665072 . \mathrm{html}$ (accessed $21 \mathrm{Jan}$ 2017).

24. Altman DG. Statistics in medical journals: some recent trends. Stat Med 2000;19:3275-89.

25. De Vuyst $\mathrm{H}$, Mugo NR, Chung MH, et al. Prevalence and determinants of human papillomavirus infection and cervical lesions in HIV-positive women in Kenya. Br J Cancer 2012;107:1624-30.

26. Zeier MD, Botha MH, Engelbrecht S, et al. Combination antiretroviral therapy reduces the detection risk of cervical human papilloma virus infection in women living with HIV. AIDS 2015;29:59-66.

27 Kelly HA, Sawadogo B, Chikandiwa A, et al. Epidemiology of high-risk human papillomavirus and cervical lesions in African women living with HIV/AIDS: effect of anti-retroviral therapy. AIDS 2017;31:273-85.

28 Rositch AF, Gravitt PE, Tobian AA, et al. Frequent detection of HPV before and after initiation of antiretroviral therapy among HIV/HSV-2 co-infected women in Uganda. PLoS One 2013;8:e55383.

29 Memiah P, Mbuthia W, Kiiru G, et al. Prevalence and risk factors associated with precancerous cervical Cancer lesions among HIV-Infected women in Resource-Limited Settings. AIDS Res Treat 2012;2012:1-7.

30 Mogtomo ML, Malieugoue LC, Djiepgang C, et al. Incidence of cervical disease associated to HPV in human immunodeficiency infected women under highly active antiretroviral therapy. Infect Agent Cancer 2009;4:9.

31 Ezechi OC, Ostergren PO, Nwaokorie FO, et al. The burden, distribution and risk factors for cervical oncogenic human papilloma virus infection in HIV positive Nigerian women. Virol $J$ 2014;11:5.

32 Ononogbu U, Almujtaba M, Modibbo F, et al. Cervical Cancer risk factors among HIV-infected Nigerian women. BMC Public Health 2013;13:582.

33 Huchko MJ, Leslie H, Sneden J, et al. Risk factors for cervical precancer detection among previously unscreened HIV-infected women in western Kenya. Int J Cancer 2014;134:740-5.

34 McKenzie KP, Rogers RK, Njoroge JW, et al. Cervical squamous intraepithelial lesions among HIV-positive women on antiretroviral therapy in Kenya. Curr HIV Res 2011;9:180-5.

35 Bekolo CE, O'Bryan G, Tchago FE, et al. Integrating cervical Cancer screening with HIV care in Cameroon: comparative risk analysis of cervical disease in HIV-Infected women receiving antiretroviral therapy to women in the General Population. PLoS One 2016;11:e0149152.

36 Mwakigonja AR, Torres LM, Mwakyoma HA, et al. Cervical cytological changes in HIV-infected patients attending care and treatment clinic at Muhimbili National Hospital, Dar es Salaam, Tanzania. Infect Agent Cancer 2012;7:3.

37 Firnhaber C, Westreich D, Schulze D, et al. Highly active antiretroviral therapy and cervical dysplasia in HIV-positive women in South Africa. $J$ Int AIDS Soc 2012;15:17382.

38 Omar T, Schwartz S, Hanrahan C, et al. Progression and regression of premalignant cervical lesions in HIV-infected women from Soweto: a prospective cohort. AIDS 2011;25:87-94.

39 Adler DH, Kakinami L, Modisenyane T, et al. Increased regression and decreased incidence of human papillomavirus-related cervical lesions among HIV-infected women on HAART. AIDS 2012;26:1645-52.

40 Capo-Chichi CD, Aguida B, Chabi NW, et al. Diversity of high risk human papilloma viruses in women treated with antiretroviral and in healthy controls and discordance with cervical dysplasia in the South of Benin. Infect Agent Cancer 2016;11.

41 Gedefaw A, Astatkie A, Tessema GA. The prevalence of precancerous cervical Cancer lesion among HIV-infected women in southern Ethiopia: a cross-sectional study. PLoS One 2013;8:e84519.

42 Huchko MJ, Leslie H, Sneden J, et al. Risk factors for cervical precancer detection among previously unscreened HIV-infected women in western Kenya. Int J Cancer 2014;134:740-5.

43 Ononogbu U, Almujtaba M, Modibbo F, et al. Cervical Cancer risk factors among HIV-infected nigerian women. BMC Public Health 2013;13:582. 
44 Jaquet A, Horo A, Ekouevi DK, et al. Risk factors for cervical intraepithelial neoplasia in HIV-infected women on antiretroviral treatment in Côte D'ivoire, West Africa. PLoS One 2014;9:e90625.

45 Mutyaba I, Phipps W, Krantz EM, et al. A Population-Level evaluation of the effect of antiretroviral therapy on Cancer incidence in Kyadondo County, Uganda, 1999-2008. J Acquir Immune Defic Syndr 2015;69:481-6.

46 Balestre E, Ekouevi DK, Tchounga B, et al. Immunologic response in treatment-naïve HIV-2-infected patients: the leDEA West Africa cohort. J Int AIDS Soc 2016;19:20044.

47 Nanda K, McCrory DC, Myers ER, et al. Accuracy of the Papanicolaou test in screening for and follow-up of cervical cytologic abnormalities: a systematic review. Ann Intern Med 2000;132:810-9.
48 Huchko MJ, Sneden J, Sawaya G, et al. Accuracy of visual inspection with acetic acid to detect cervical Cancer precursors among HIV-infected women in Kenya. Int J Cancer 2015;136:392-8.

49 Bratcher LF, Sahasrabuddhe VV. The impact of antiretroviral therapy on HPV and cervical intraepithelial neoplasia: current evidence and directions for future research. Infect Agent Cancer 2010;5:8.

50. Hawes SE, Critchlow CW, Faye Niang MA, et al. Increased risk of high-grade cervical squamous intraepithelial lesions and invasive cervical cancer among African women with human immunodeficiency virus type 1 and 2 infections. J Infect Dis 2003;188:555-63.

51. Anastos K, Hoover DR, Burk RD, et al. Risk factors for cervical precancer and cancer in HIV-infected, HPV-positive Rwandan women. PLoS One 2010;5:e13525. 